\title{
Levitation of an iron ball in midair without active control
}

\author{
S. Sasaki \\ Iwate High School, Nagatacho 7-60, Morioka, 020-0062, Iwate, Japan \\ I. Yagi \\ Department of Education, Iwate University, Ueda 3-18-33, Morioka, 020-8550, Iwate, Japan \\ M. Murakamia \\ Department of Materials Science and Engineering, Shibaura Institute of Technology Shibaura 3-9-14, \\ Minato-ku, Tokyo 108-8548, Japan
}

(Received 22 September 2003; accepted 20 November 2003)

\begin{abstract}
An iron ball floats in midair in a plastic box when several iron balls were attracted by a permanent magnet. A complex interaction between magnetized sphere materials and a lifting magnet enabled the suspension of an iron ball. The balls in the first row are simply attracted by the lifting magnet. The ball in the second row is also attracted by the lifting magnet, however, due to the repulsive forces exerted from the balls sitting above, it can float in midair. We also found that there are two stable positions for the ball to float. The floating ball could be transported from one equilibrium position to another by simply rotating the lifting magnet. This will make it possible to construct a noncontact load transport device. (c) 2004 American Institute of Physics.
\end{abstract}

[DOI: $10.1063 / 1.1640788]$

\section{INTRODUCTION}

Levitation of objects is fascinating and has always attracted worldwide interest. In fact many people devised magnetic levitation in that permanent magnets and superconductors levitated or floated or were suspended in midair. ${ }^{1,2}$ It is known that there are several ways to magnetically levitate objects. ${ }^{3}$ Attraction between a permanent magnet and a piece of iron causes the iron to levitate, although the floating iron must be constrained or supported by something. Attraction between an electromagnet and a piece of iron has also been used to levitate the iron. ${ }^{4}$ But for stable levitation, one needs to actively control the current supplied to the electromagnet in order to maintain some distance between them by carefully monitoring the distance with sensors. ${ }^{4}$

It is now commonly accepted that stable levitation of permanent magnets in static field is impossible, which is known as Earnshaw's theorem. ${ }^{5}$ In this report we show that one can levitate an iron ball without active control with the help of other magnetized iron balls.

\section{EXPERIMENT}

We used an $\mathrm{Fe}-\mathrm{Nd}-\mathrm{B}$ ring magnet $50 \mathrm{~mm}$ in outer diameter, $30 \mathrm{~mm}$ in inner diameter, and $40 \mathrm{~mm}$ in height to attract iron balls about $10 \mathrm{~mm}$ in diameter and $4 \mathrm{~g}$ in weight. The ring magnet is magnetized along its central axis. Twelve iron balls are stored in a plastic box $60 \mathrm{~mm}$ in length, 12.5 $\mathrm{mm}$ in width, and $50 \mathrm{~mm}$ in height. The thickness of the plastic board was $1 \mathrm{~mm}$. The magnet was fixed by glue tape on the box as its pole axis tilted $90^{\circ}$ from a horizontal line and perpendicular to the wide walls of the plastic box. We then shook the plastic box with the magnet firmly fixed on

${ }^{a)}$ Electronic mail: masatomu@sic.shibaura-it.ac.jp the box. Shaking made the iron balls to bounce in the box. After shaking, some iron balls are attracted by the lifting magnet. We then observed how the iron balls are distributed inside the box. We also rotated the lifting magnet and observed how the arrangement of iron balls changed.

\section{RESULTS AND DISCUSSION}

Figure 1 shows one pattern of the arrangement of iron balls attracted by the lifting magnet, where the direction of magnetization of the magnet is perpendicular to the row of iron balls. Here five iron balls in the first row were simply attracted by the magnet with all the balls in contact with the ceiling board. It is, however, notable that an iron ball in the second row floated in midair without contact. One can see that the position for the ball to float is just below the iron ball situated at the center of the lifting magnet. Since we are using a permanent magnet, we cannot actively control the field strength and the distance. Furthermore, once the ball sat at this stable position, it stayed there unless we changed the field configuration. We left the ball floating for three days and found it stayed there. The present experimental fact shows that there is a position for the ball to float where the force balance conditions are fulfilled both in vertical and horizontal directions.

In order to stabilize the levitation of an iron ball, however, some repulsive force must be incorporated in addition to the attractive force from the lifting magnet. We believe that the iron ball sitting above the floating ball is the main source of the repulsive force. In the present magnet field configuration, the magnetic field lines are almost parallel to the horizontal line in the vicinity of the magnet center. Hence, two iron balls are magnetized along the same horizontal direction. Suppose that the magnetization values of two balls are $m_{1}$ and $m_{2}$ with respective heights of $r_{1}$ and $r_{2}$ 


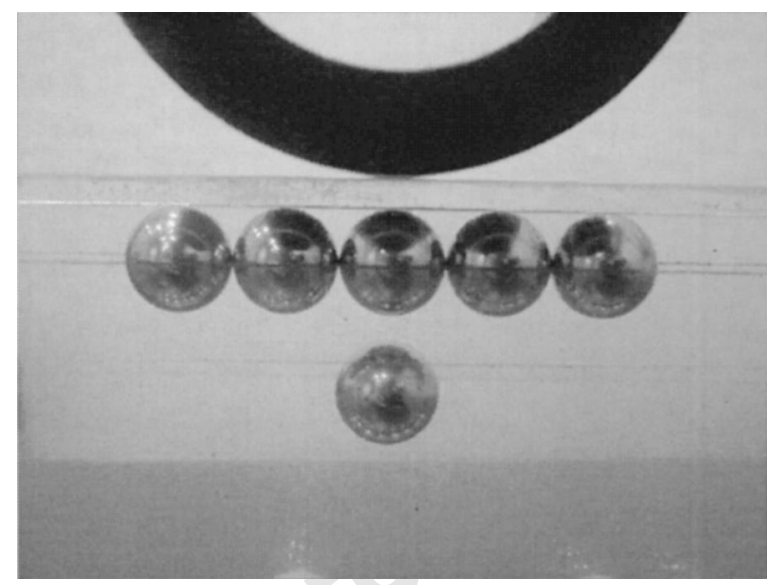

FIG. 1. Photograph of an iron ball floating in midair.

from the ground, and the magnetization and the height of the lifting magnet are $M$ and $H$. Then the attractive force between the magnet and the floating ball is given by

$$
F_{M 2}=\frac{3 M m_{2}}{4 \pi \mu_{0}\left(H-r_{2}\right)^{4}},
$$

where $\mu_{0}$ is the permeability of vacuum. Since two iron balls are magnetized in the same direction, a repulsive force

$$
F_{12}=\frac{3 m_{1} m_{2}}{4 \pi \mu_{0}\left(r_{2}-r_{1}\right)^{4}}
$$

will act on two balls, which stabilizes the levitation. The force balance in a vertical direction is then given by

$$
F_{M 2}=F_{12}+m g,
$$

where $m$ is the mass of the floating ball and $g$ is the acceleration of gravity. Here $F_{M 2}$ and $F_{12}$ are the functions of the heights of two balls, and there will be a range in the height for the levitation ball to be stable. In fact we could see that the levitating ball bounced when the plastic box was slightly shook.

This is similar to the situation that two like-pole magnets are placed in parallel, which will inhibit two balls from approaching each other, although the lifting magnet is exerting attractive forces toward two balls, which is schematically illustrated in Fig. 2.

For stable levitation, the force balance must also be met along the horizontal direction. In the present configuration, since the balls are aligned along the center plane of the magnetic field generated from the lifting magnet, the balls will be autostable along the horizontal direction. In other words, they try to stay centered in the field of a large magnet. In addition, as schematically shown in Fig. 2, two neighboring balls sandwiching the ball sitting above will also exert repulsive forces on the floating ball such that it is repelled toward the center position. Thus, the floating ball is stable at the center in the horizontal direction.

Even in this case, the question is raised for the stability along the direction perpendicular to this direction on the same horizontal plane. Since the iron ball is located at the center axis of the magnet, it may stay centered. However, such three-dimensional stable levitation is forbidden by

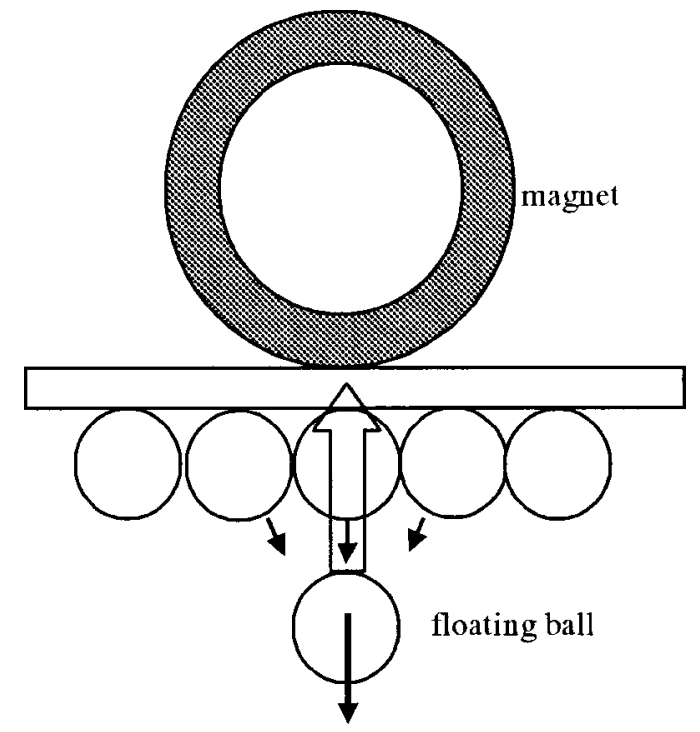

FIG. 2. Schematic illustration of the configuration of iron balls and forces, which enabled the levitation of an iron ball. The lifting magnet attracts the floating ball, while three balls in the first row exert repulsive forces. Here the direction of the magnetization of the ring magnet is parallel to its central axis.

Earnshaw's theorem. ${ }^{5}$ One may then suspect that the levitating ball touches the plastic wall. Figure 3 shows a photograph of the levitating iron ball sandwiched by the plastic walls. One can see that the ball does not touch the wall. The fact that the floating ball bounced at this position also supports that the ball floats without touching the wall. It is then probable that the plastic wall has a small diamagnetic susceptibility and exerts a repulsive force to stabilize the levitation. ${ }^{6}$

Through several trials we also found that there is another stable position for the ball to levitate as shown in Fig. 4. In this case, the ball in the first row is not situated at the magnet center, instead two balls sat with their center being the magnet center. Here, one can again think that three iron balls are magnetized in the same direction due to the field generated from the lifting magnet. The lifting magnet attracts the floating ball, however, two balls sitting above will exert repulsive

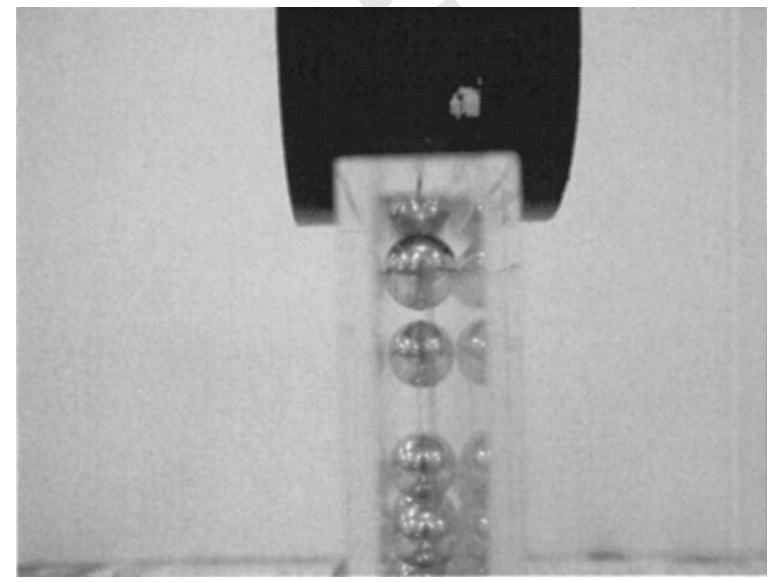

FIG. 3. Side view of a floating iron ball. It floats in between two plastic walls. 


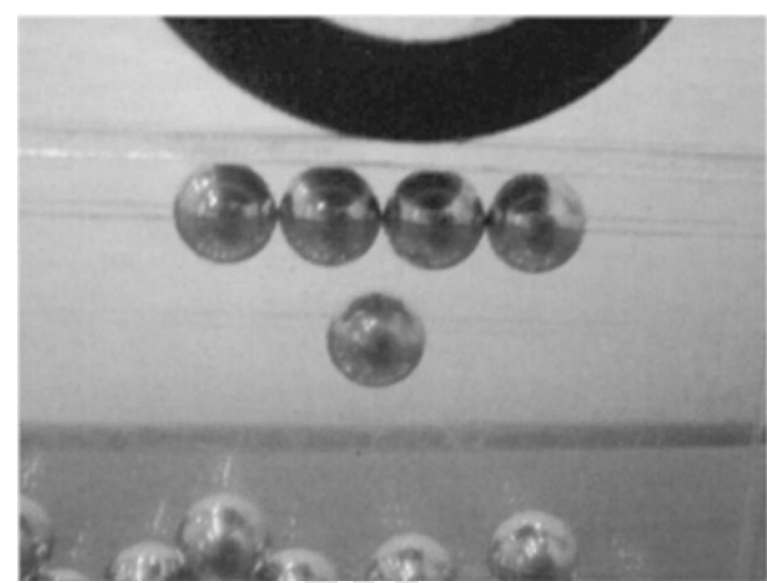

FIG. 4. Photograph of an iron ball floating in midair. There is another stable position for the ball to float.

forces. This made it possible to levitate an iron ball in the position schematically shown in Fig. 5. As a result, the ball in the second row can float in midair. Like the case in Fig. 1, two balls sitting above will exert the restoring force for the floating ball to be centered to the equilibrium position along the horizontal direction, which is also sketched in Fig. 4.

It was interesting that we could transport this floating ball from one equilibrium position to another by simply rotating the lifting magnet. Figure 6 shows the photo of the ball leaving the position below the ball sitting at the magnet center to the next stable position. Such a process is schematically illustrated in Fig. 7. This result shows that there is a continuous route or a continuous potential well for the ball to float. This fact suggests that one can transport a load without contact using the present configuration of iron balls, which provides the possibility of a noncontact load transport device.

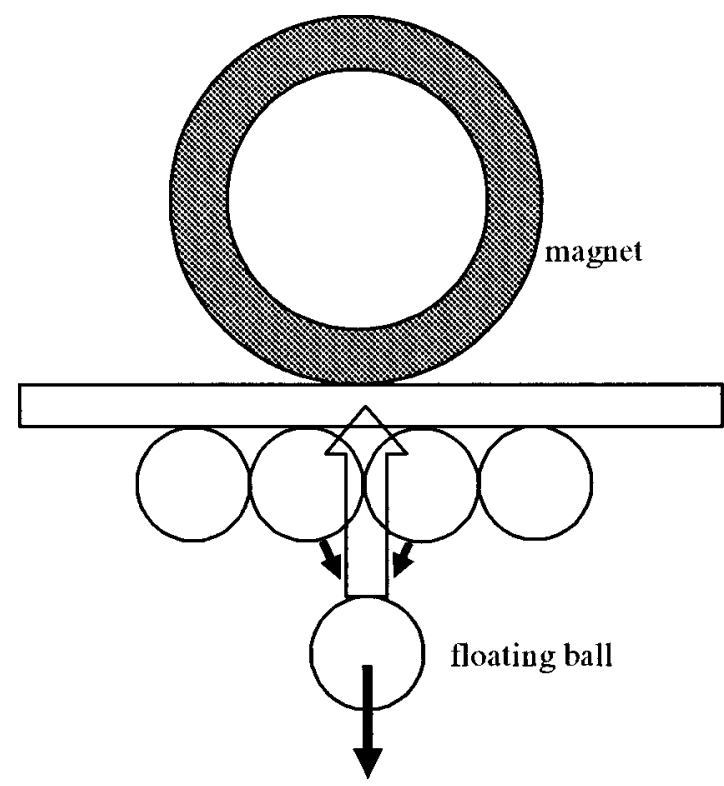

FIG. 5. Schematic illustration of the configuration of iron balls and force, which enabled the levitation of an iron ball. The lifting magnet attracts the floating ball, while two balls sitting above exert repulsive force.

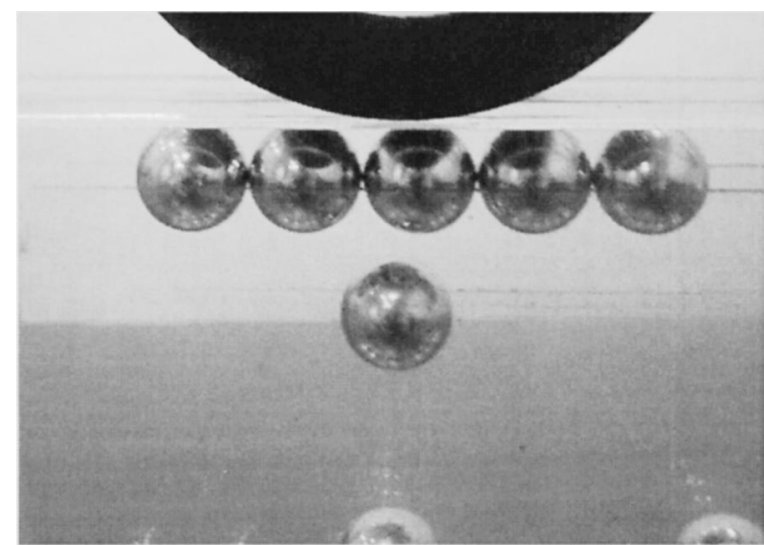

FIG. 6. Photograph of an iron ball trying to escape from one stable position to another when the lifting magnet was rotated.

Here all the experiments were performed in a plastic box, thus the levitation of iron balls was not realized in free space. Nevertheless, the present experiment shows interesting features of sphere magnetic materials along with the fact that at least with the help of plastic walls we can suspend an iron ball in midair and can transport it without contact.

\section{SUMMARY}

We have shown that an iron ball floats in midair without any active control. This is probably due to the interaction of several iron balls. In the case of sphere magnetic materials, they can be magnetized in the same direction without close contact, which may be the source of repulsive interaction stabilizing the force balance along a vertical direction. Horizontal stabilization is achieved by positioning the ball at the magnet center at least in one direction. The stabilization in

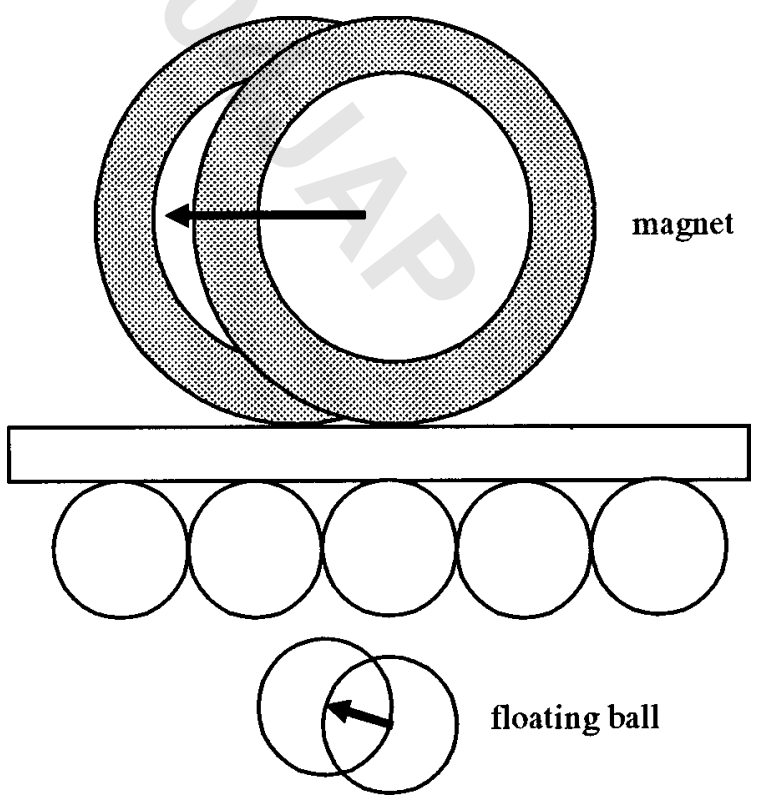

FIG. 7. Schematic illustration of the process for the floating ball to move from one stable position to another. 
the perpendicular direction is not clear at present. It is probable that small diamagnetic susceptibility of the plastic board may help the stability. It was also found that there are two stable positions for the iron ball to levitate. The ball could be transported from one equilibrium position to another by simply rotating the lifting magnet.
${ }^{1}$ M. Murakami et al., Jpn. J. Appl. Phys., Part 2 29, L1991 (1990).

${ }^{2}$ E. H. Brandt, Science 243, 349 (1989).

${ }^{3}$ http://my.execpc.com/ rhoadley/maglev.htm.

${ }^{4}$ J. Cicon, Popular Electronics 78, 48 (1996).

${ }^{5}$ S. Earnshaw, Trans. Cambridge Philos. Soc. 7, 97 (1842).

${ }^{6}$ A. K. Geim, M. D. Simon, M. I. Boamfa, and L. O. Heflinger, Nature (London) 400, 323 (1999). 